\title{
MESA: A portable multi-energy sensor array for low-frequency electromagnetic field fluctuations
}

\author{
JAMES HOURAN, RENSE LANGE, and DAVID L. BLACK \\ University of Illinois, Springfield, Illinois
}

\begin{abstract}
The study of the relation between electromagnetic fields and various psychological phenomena would be enhanced by the measurement and correlation of time series over multiple energy bands. The paper discusses the design and implementation of a computerized multi-energy sensor array (MESA) that detects and records low-frequency energy fluctuations. The current implementation of MESA focuses on the measurement of magnetic fields, infrared and visible light frequencies, and indices of seismic activity. However, MESA is sufficiently flexible to accommodate the measurement of other variables as well, and it can be configured to suit applications in laboratory and field settings.
\end{abstract}

Because electromagnetic fields (EMFs) are associated with hazardous biological and behavioral changes, their detection and measurement are an important issue (see, e.g., Kristensen, 1989; Persinger, Ludwig, \& Ossenkopp, 1973; Wiedemann \& Schultz, 1995). Whereas most research emphasizes man-made sources of EMFs, such as power lines (e.g., Savitz, 1993), the effects of increased geomagnetic activity have received less widespread attention. This is unfortunate, since increases in geomagnetic activity are correlated with medical conditions such as sudden infant death syndrome (R. P. O'Connor \& Persinger, 1997) and immunological alterations (Persinger, 1983).

Geomagnetic activity is also associated with more benign effects, such as the onset of hallucinatory episodes and other abnormal behaviors (e.g., Gearhart \& Persinger, 1986; Persinger, 1988, 1993; Persinger \& Cameron, 1986; Randall \& Randall, 1991). It has been hypothesized (Persinger, 1988, 1993) that an increase in geomagnetic activity alters melatonin and serotonin levels in the temporal lobe, leading to microseizures. The accompanying hallucinations and perceptions may involve vestibular alterations, auditory sequences, odd smells, visual forms, acute emotional responses, and disruption from sleep (particularly during REM periods) (Baldwin, 1970; Sterman, Shouse, \& Passouant, 1982). It has further been found that these effects closely resemble sensations reported by individuals whose temporal lobe structures are stimulated by focused, extremely low-frequency electric currents (Persinger \& Cameron, 1986).

The research cited above characteristically samples only a limited number of electromagnetic frequencies. Savitz (1993), however, has argued that the interactions among

J.H. is currently at the SIU School of Medicine, Springfield, IL; R.L. is currently with the Illinois State Board of Education, Springfield, IL; and D.L.B. is currently at JAM Software, Inc., Cupertino, CA. We thank Ronald A. Havens, Dean I. Radin, Michael A. Persinger. Stan Koren, and Reuven Sandyk for comments on an earlier draft of this paper. Correspondence should be addressed to J. Houran, 301 University Court West, Springfield, IL 62703 or to R. Lange (e-mail: rlange3726@ aol.com). the various frequencies of the electromagnetic spectrum may be of crucial importance, considering the notably different effects of ionizing radiation, microwaves, and visible light on physiological functioning. Consequently, it seems desirable to study the physiological effects associated with the interactions of multiple frequencies over time.

This paper describes a multi-energy sensor array (MESA), capable of sampling and recording several different frequencies over time so that they can be correlated with standard signal-processing techniques (e.g., Stearns \& David, 1988). The current version of MESA was designed to investigate Persinger's hypotheses related to geomagnetic activity. For this reason, the presentation emphasizes the measurement of magnetic fields, infrared (IR) and visible light frequencies, and seismic activity. We want to emphasize, however, that MESA's design is sufficiently flexible to accommodate the measurement of other EMFs as well, and that it can be configured to suit the needs of applications in laboratory and field settings.

\section{MESA \\ System and Support Specifications}

As is shown in Figure 1, the current implementation of MESA can accommodate a maximum of eight sensor inputs that lead into a data acquisition board that is controlled by a portable minicomputer with a moderately sized hard disk drive. Naturally, a phone link can be used between experiments to download the data to a larger computer system with greater secondary storage capacity.

The technical specifications for MESA are listed in Tables 1 and 2 for quick reference. Here we will describe the sensors used in the current implementation of MESA, together with their functional properties.

\section{Sensors}

Although MESA can accommodate up to eight sensor channels simultaneously, only five are used currently.

Channels 1 \& 2: Tri-Field meters (AlphaLab, Inc.) can detect three fields--magnetic, electric, and radio fre- 


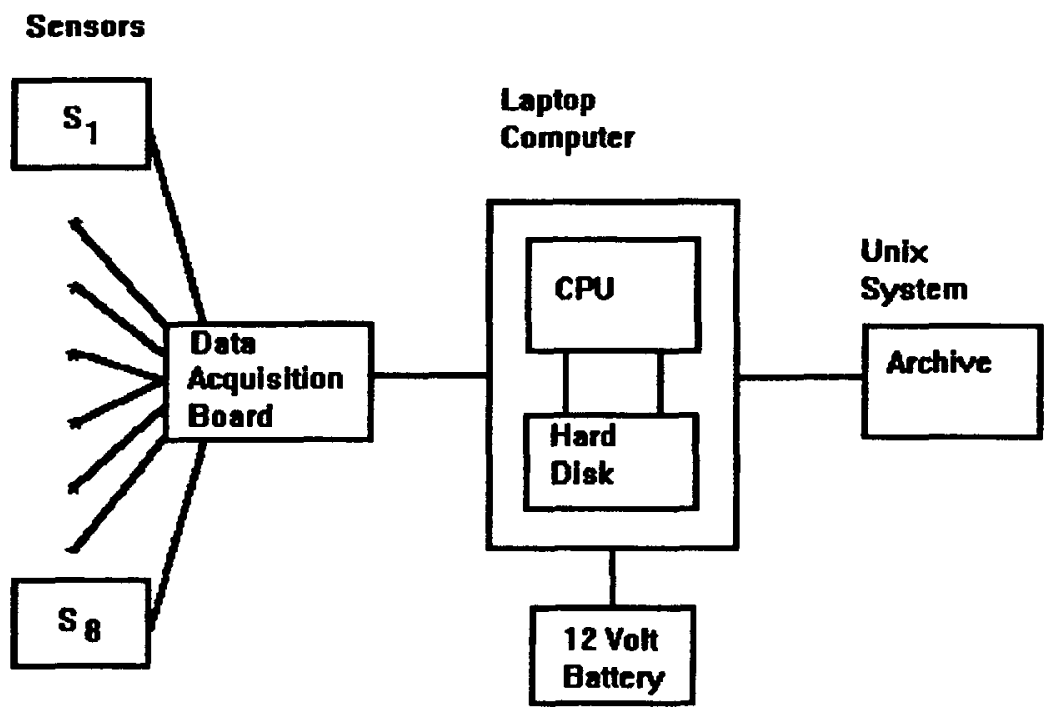

Figure 1. An outline of MESA.

quencies. However, the three fields cannot be sampled simultaneously. The meters have two ranges for magnetic fields, $0-3 \mathrm{mG}$ and $0-100 \mathrm{mG}$. These meters measure the RMS value of sinusoidal magnetic fields. This is a limitation of the device, which is designed to measure ac magnetic fields from 0.2 to $100 \mathrm{mG}$ at $60-\mathrm{Hz}$ frequency. The output voltage for the meter ranges from $0.89 \mathrm{~V}$ for a "zero" indication, to approximately $2.55 \mathrm{~V}$ for a fullscale indication. Calibration tests have shown that this output voltage remains proportional to the external field strength even past the meter's ability to visually indicate the field strength (i.e., the meter was "pegged").

Channel 3: A CdS photoresistor (Radio Shack, No. 276-1657) operates in the variable resistance mode and is sensitive to visible light wavelengths. The frequency/ sensitivity curve for CdS is very close to that of the human eye. Its time domain response characteristic is variable; that is, its output depends on how quickly the intensity of a light source is changing. Minor variations appear to be tracked precisely. For instance, the $60-\mathrm{Hz}$ flicker of a fluorescent light is easily seen on the output of this detector. However, large changes can require up to $2 \mathrm{sec}$ to be accurately reflected in this sensor. The sensor is such that a high voltage results from low ambient light conditions, and a low voltage results from high ambient light conditions. A pull-up resistor is selected which nets the desired sensitivity.

Channel 4: The infrared photoresistor (Radio Shack, No. 276-145) consists of a single transistor whose base region is sensitive to IR wavelengths. Connections to the base and emitter terminals of the transistor make it possible to use it as a variable-resistance device, similar to the $\mathrm{CdS}$ photoresistor mentioned above. The IR transistor's characteristics with regard to light levels are very similar to those of the CdS device, with two exceptions: (1) The IR sensor is most sensitive in the infrared (long- wavelength light) regions, and (2) the response of the IR sensor is much faster, in the nanosecond range. Calibration tests revealed that a $60-\mathrm{Hz}$ flicker source is very easily seen on the IR's output, and response to light changes is virtually instantaneous.

Channel 5: The Vibration/relative seismic activity sensor (Radio Shack, No. 273-091) is a large piezoelectric transducer, which is sold as a speaker element. By mechanically coupling the device to a suitable structure, such as a door frame or floor, it functions as a vibration sensor. Voltages as high as $+11 \mathrm{~V}$ were measured from the device with moderate levels of compression. High levels of compression presented a problem for the A/D equipment, because overloading of a $+5-\mathrm{V} \mathrm{A} / \mathrm{D}$ input resulted in "splatter" across adjacent channels. However,

Table 1

Technical Specifications for MESA

\begin{tabular}{ll}
\hline Number of channels & 8 \\
Sampling resolution & 8 bits (256 discrete values) per channel \\
Sampling frequency & Variable: $1-40,60$, and \\
& 1,440 samples/sec \\
& $0.0-5.0 \mathrm{~V}$ (each channel) \\
Input voltage & $>20 \mathrm{M} \Omega$ (each channel) \\
Input impedance & $+12 \mathrm{~V} \mathrm{dc}$ \\
Voltage supply requirement & \\
Current supply requirement & $700 \mathrm{~mA}$ (computer), 10 mA (A/D unit) \\
Power requirement & $9 \mathrm{~W}$ \\
Sensor types supported & Variable voltage (chs. 1-8), Variable \\
& resistance (chs. 1-4) \\
Input connector type & $50 \Omega$ BNC \\
Time base & Supplied from host computer: $100-\mathrm{Hz}$ \\
& interrupt-driven clock \\
Computer interface & Bidirectional Centronics--compatible \\
& parallel port (TTL) \\
Digital I/O (computer view) & A0-A2 (output): channel select \\
& Data (input): serial data from A/D \\
& converter \\
& Clock (output): sample start signal and \\
& data clock \\
\hline
\end{tabular}


Table 2

MESA Data Storage Format: Variable-Length Packets

\begin{tabular}{|c|c|}
\hline Byte & Contents \\
\hline $\begin{array}{l}{[7]=1} \\
{[7]=0} \\
{[6: 4]} \\
{[3: 0]}\end{array}$ & $\begin{array}{l}\text { Packet contains timestamp } \\
\text { Packet does not contain timestamp } \\
\text { Reserved } \\
\text { No. of one-byte samples to follow }\end{array}$ \\
\hline $\begin{array}{l}\text { (time-st } \\
2-5 \\
6-n\end{array}$ & $\begin{array}{l}\text { amped packet) } \\
\text { Four-byte time stamp: } \\
\text { long integer, Intel byte ordering-seconds } \\
\text { elapsed since 00:00:00 GMT, January 1, } 1970 \\
\text { channel data, lowest numbered channel first }\end{array}$ \\
\hline
\end{tabular}

this problem was solved by placing a $5-\mathrm{V}$ Zener diode across the piezo's terminals so that any output voltages higher than the Zener would shunt to ground.

\section{Support Parts and Sources}

All pull-up switches (RS1-RS4) used in MESA are composed of 6PDT rotary switches from Radio Shack (No. 275-1386) and multivalued resistors pulling up the sensor inputs to $+5 \mathrm{~V}$. All resistors are a $0.25-\mathrm{W}, 5 \%$ tolerance, carbon film type (JDR Microdevices, RA30). The project box (i.e., instrumentation case) is a JDR, GPB-862. In addition, $50-\Omega$ BNC chassis connectors (JDR BNCF), a $7805+5-V$ regulator (JDR, 7805T), and a parallel port connector (to computer) (JDR, DB25S) are used. MESA currently uses coaxial cables (RD58U), approximately $25 \mathrm{ft}$ in length, for connection of the sensors to the $\mathrm{A} / \mathrm{D}$ converter.

\section{Placement of the Sensors}

Figure 2 illustrates the placement of the aforementioned sensors for our research purposes. Optionally, a $2 \times 2 \mathrm{ft}$ Plexiglas sheet may be used to affix the sensors (i.e., the two Tri-Fields and the two light sensors) in a fixed position by means of Velcro or electrical tape. This arrangement facilitates their mobility, since all secured sensors may now be moved simultaneously. However, the piezoelectric transducer is bonded to the floor, ground, or other stable surface.

\section{Data Acquisition Board}

MESA's data acquisition board comprises an eightchannel analog multiplexer (JDR, 4051) and a serial A/D converter (JDR, ADC0831) with 8-bit resolution $( \pm 0.5 \mathrm{LSB})$ and maximum $32-\mu \mathrm{sec}$ conversion time. The first four inputs to the analog multiplexer are wired to 6PDT rotary switches, with the first five switch positions causing the multiplexer inputs to be connected to resistive pullups to the $+5-\mathrm{V}$ rail: $1 \mathrm{~K}, 4.75 \mathrm{~K}, 10 \mathrm{~K}, 100 \mathrm{~K}$, and $1 \mathrm{M} \Omega$. The sixth (rightmost) position of the rotary switch provides no pull-up voltage. The remaining four inputs to the analog multiplexer (channels 5-8) are wired directly to the multiplexer's inputs, for use with variable-voltage sensors only.

The computer $A / D$ unit interface is via the computer's parallel port, utilizing TTL voltages $(0$ and $+5 \mathrm{~V})$. Three pins are outputs (A0-A2) and select which of the eight of the analog mulitplexer's inputs are to be connected to the ADC's input. One pin is the clock output, which signals the start of a sample to the ADC and also clocks data out of the ADC. The data input supplies serial data from the ADC to the computer, clocked out one bit at a time, by the clock output, as mentioned above.

Although the eight channels are sampled sequentially, this occurs so quickly that the process appears simultaneous. In particular, the skew between the channels can hardly be detected for rates up to 40 samples/sec. The exact sample rate may vary somewhat over a session, because the computer's operating system Linux features multiprocessing, which may occasionally cause the CPU to be diverted from the task of sampling. It was found, however, that the variations in the sampling rate were insignificant when no contention for processor resources existed.

Unconnected analog inputs were pulled up by a 1-M $\Omega$ resistor to $+5 \mathrm{~V}$, to minimize channel crosstalk. The ana$\log$ multiplexer (and wires leading to its inputs) exhibit some capacitive effects, which causes signal levels from

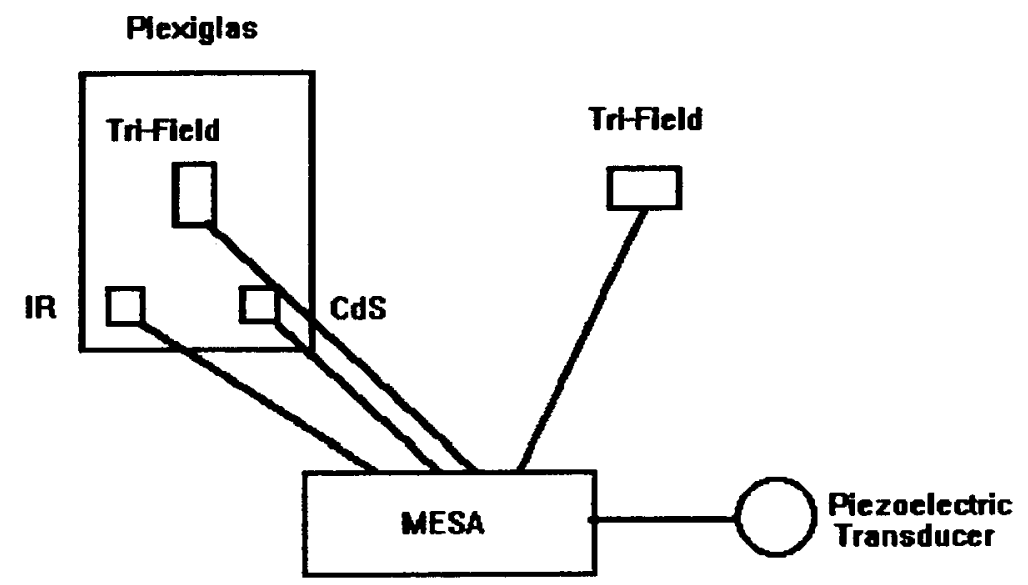

Figure 2. Sensor arrangement in current implementation of MESA. 
previous channels to be held if not actively driven by a signal source or resistive pull-up. This prevents the appearance of "shadowing" effects or signal changes in adjacent channels following signal changes in an active channel. With sufficient strength pull-up (or the presence of another active signal source), the effects of shadowing can be completely nullified. Note that shadowing occurs only in completely unconnected channels.

\section{The Computer System}

MESA runs on a sub-notebook computer featuring an Intel 80486 SLC 33-MHz processor with $4 \mathrm{MB}$ of RAM and an 85-MB hard drive, using the Linux operating system. All software is written in Gnu C compiler and SVGAlib - a package of display primitives for the VGA display most commonly found on IBM-PC-compatible systems. The supplied software utilizes the $640 \times 480$ $\times 1$ bit plane mode to display sampled data in "real-time" (i.e., while the experiment is in progress) as well as in "playback" mode (i.e., after the data have been gathered). At an acquisition speed of 40 samples/sec, MESA's storage requirement is 464 bytes $/ \mathrm{sec}$, or approximately $1.3 \mathrm{MB} / \mathrm{h}$. Other features, such as variable playback speed and magnification of the displays, are also implemented. Most importantly, variable alarm levels can be specified for any one of the eight possible sensor channels. For example, MESA currently uses a reading of $\geq 7 \mathrm{mG}$ on both of the Tri-Field meters over a 3-sec interval as a threshold to trip the computerized alarm.

\section{Power}

MESA is self-contained, including the power supply. Therefore, the various components (i.e., acquisition board, computer, and most of the sensors) are powered by a 10 lb, 12-V deep-discharge marine battery of $34-\mathrm{AH}$ capacity. The battery is connected to a positive voltage regulator (JDR, 7805T) which produces exactly $5 \mathrm{~V}$, provided that the battery voltage remains at or above $+5 \mathrm{~V}$. The calibration tests on all of MESA's components and sensors indicated that the apparatus can be run for over $12 \mathrm{~h}$ continuously on this battery before recharging is required.

\section{Archive}

Upon the completion of an experiment, the observational data can be transferred from the laptop computer for analysis by two methods. The first is the use of a pocket Ethernet adapter to transfer the data using file transfer protocol (ftp) or other protocol utilizing a TCP/IP networking infrastructure. In the prototype, a TCP/IP stack for Workgroups 3.11 was installed on another computer, along with an Ethernet card, and supplied Telnet and ftp clients on the machine running Windows for Workgroups were used to transfer recorded data. The second method is to log on to the laptop computer via its serial port and download data using a transfer protocol such as $\mathrm{Zmodem}$.

\section{DISCUSSION}

This paper showed that it is feasible to construct a multi-energy sensor array that is portable and relatively inexpensive (the current implementation costs approximately $\$ 3,000$ ) and that can be operated by a single researcher. Thus, it becomes practical to obtain simultaneous time series over multiple EMF bands not only in the laboratory (see, e.g., Green, Parks, Guyer, Fahrion, \& Coyne, 1992; Radin \& Rebman, 1996) but also in natural settings. This approach may be especially pertinent to studying the role of EMFs in instigating hallucinations and other psychophysiological effects, since various series of frequency interactions may elicit notably different behavioral responses. The authors are currently using MESA in field studies to extend the research correlating EMFs with these hallucinations and abnormal behaviors. However, research in this area might also provide insights into the role EMFs have in such diverse areas as dream recall (Sandyk, 1995), correction of regulatory dysfunctions (Vasilevskii, Sidorov, \& Suvorov, 1993), rearing behavior in rats (Rudolph, Krauchi, Wirz, \& Feer, 1985), and physical and psychological therapies (M. E. O'Connor, 1993).

Although it would certainly be possible to construct a superior system by using more advanced components, calibration tests indicated that MESA achieved the reliability and precision required for our research. In addition, the option to substitute different sensors makes MESA sufficiently flexible for use in a variety of settings.

\section{REFERENCES}

BaLDWIN, M. (1970). Neurological syndromes and hallucinations. In W. Keup (Ed.), Origin and mechanisms of hallucinations (pp. 3-12). New York: Plenum.

Gearhart, L., \& Persinger, M. A. (1986). Geophysical variables and behavior: XXXIII. Onset of historical poltergeist episodes with sudden increases in geomagnetic activity. Perceptual \& Motor Skills, 62, 463-466.

Green, E., Parks, P., Guyer, P. M., Fahrion, S. L., \& Coyne, L. (1992). Gender differences in a magnetic field. Subtle Energies, 3, 65-103.

KRISTENSEN, T. S. (1989). Cardiovascular diseases and the work environment: A critical review of the epidemiologic literature on nonchemical factors. Scandinavian Journal of Work, Environment, \& Health, $15,165-179$

O'ConNor, M. E. (1993). Psychological studies in nonionizing electromagnetic energy research. Journal of General Psychology, 120, 33-47.

O'ConNor, R. P., \& PERsinger, M. A. (1997). Geophysical variables and behavior: LXXXII. A strong association between sudden infant death syndrome and increments of global geomagnetic activitypossible support for the melatonin hypothesis. Perceptual \& Motor Skills, 84, 395-402.

PERSINGer, M. A. (1983). Geophysical variables and behavior: IX. Expected clinical consequences of close proximity to UFO-related luminosities. Perceptual \& Motor Skills, 56, 259-265.

Persinger, M. A. (1988). Increased geomagnetic activity and the occurrence of bereavement hallucinations: Evidence for melatoninmediated microseizuring in the temporal lobe? Neuroscience Letters, 88, 271-274.

Persinger, M. A. (1993). Average diurnal changes in melatonin levels are associated with hourly incidence of bereavement apparitions Support for the hypothesis of temporal (limbic) lobe microseizuring Perceptual \& Motor Skills, 76, 444-446. 
Persinger, M. A., \& Cameron, R. A. (1986). Are earth faults at fault in some poltergeist-like episodes? Journal of the American Society for Psychical Research, 80, 49-73.

Persinger, M. A., Ludwig, H. W., \& OssenkopP, K. P. (1973). Psychophysiological effects of extremely low frequency electromagnetic fields: A review. Perceptual \& Motor Skills, 36, 1131-1159.

RaDiN, D. I., \& REBMAN, J. (1996). Are phantasms fact or fantasy? A preliminary investigation of apparitions evoked in the laboratory. Journal of the Society for Psychical Research, 61, 65-87.

RANDAll, W., \& Randall, S. (1991). The solar wind and hallucinations-a possible relation to magnetic disturbances. Bioelectromag netics, 12, 67-70.

RudOlPH, K., KraUCHI, K., WIRZ, J. A., \& FEER, H. (1985). Weak 50$\mathrm{Hz}$ electromagnetic fields activate rat open field behavior. Physiol ogy \& Behavior, 35, 505-508.

SANDYK, R. (1995). Weak electromagnetic fields restore dream recall in patients with multiple sclerosis. International Journal of Neuroscience, 82, 113-125.
SAvitz, D. A. (1993). Overview of epidemiologic research on electric and magnetic fields and cancer. American Industrial Hygiene Association Journal, 54, 197-204.

STEARNS, S. D., \& DAVID, R. A. (1988). Signal processing algorithms. Englewood Cliffs, NJ: Prentice-Hall.

Sterman, M. B., Shouse, M. N., \& Passouant, P. (1982). Sleep and epilepsy. New York: Academic Press.

VASIlEVSKII, N. N., Sidorov, Y. A., \& Suvorov, N. B. (1993). Role of biorhythmological processes in mechanisms of adaptation and correction of regulatory dysfunctions. Human Physiology, 19, 5-54.

Wiedemann, P. M., \& Schultz, H. (1995). The electromagnetic fields risk issue: Constructing scenarios on further development of public debate in Germany. European Review of Applied Psychology, 45, 35-41.

(Manuscript received April 22, 1996;

revision accepted for publication May 14, 1997.) 